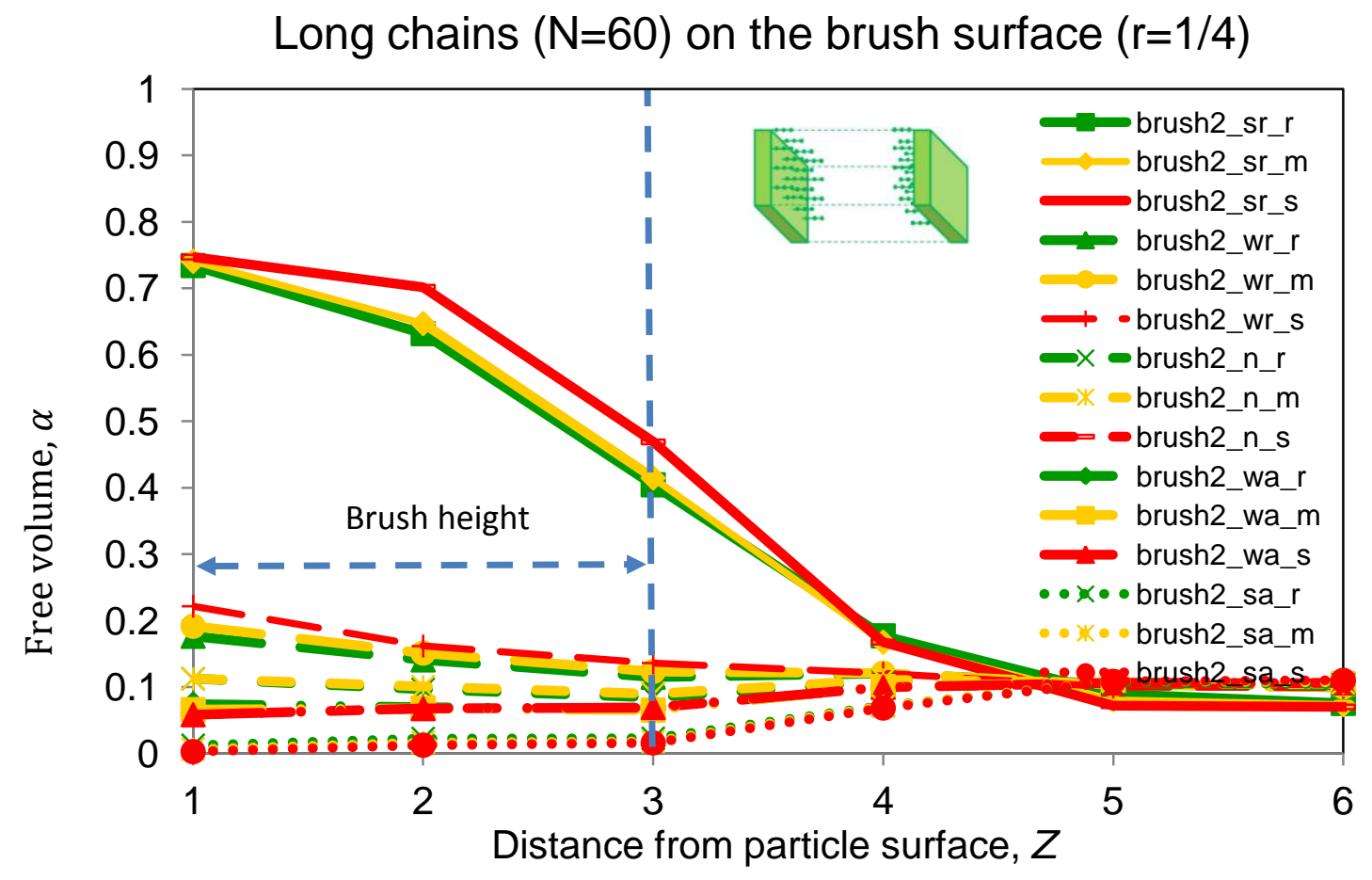




\title{
Interactions at the Interface of Polymer Matrix-Filler Particle \\ Composites
}

\author{
Jie Feng ${ }^{1,2}$, Surendar R. Venna ${ }^{1,3}$ and David P. Hopkinson ${ }^{4 *}$ \\ ${ }^{1}$ National Energy Technology Laboratory, United States Department of Energy, Pittsburgh, PA, 15236, \\ United States \\ ${ }^{2}$ Oak Ridge Institute for Science and Education, Oak Ridge, TN 378311, United States \\ ${ }^{3}$ AECOM Corporation, Pittsburgh, PA 15236, United States \\ ${ }^{4}$ National Energy Technology Laboratory, United States Department of Energy, Morgantown, WV 26507, \\ United States
}

*Corresponding author. Email address: david.hopkinson@netl.doe.gov 


\begin{abstract}
Interfacial structures of polymer matrix-filler particle composites play a crucial role in determining the properties of the composite materials, such as the gas separation performance of mixed matrix membranes. Monte Carlo simulations employing coarse-graining models show that the density distribution and the fraction of free volume of the polymer at the interface are dominated by the interaction of the polymer with the filler particle and the geometry of the filler particle surface. Here, we studied two different filler particles with smooth and brush-like (rough) surfaces and three different polymer chains with rigid, medium and soft flexibility. Five different interaction strengths between the polymers and filler particles were studied. The polymer chains deform into pancake-like conformations on the filler particle surface in all the cases. Interestingly, the polymer chains are deformed more on the surfaces of particles that have strong repulsion in comparison to attractive and neutral surfaces. Filler particles that have brush-like surfaces tend to results in larger free volume at the interface than occurs with smooth surfaced filler particles. By contrast, the rigidity of the polymer chain introduced by considering the angle potential in the coarse-grain model only slightly influences the chain conformation at the interface.
\end{abstract}

Keywords: polymer matrix composites, interface, mixed matrix membrane, fraction of free volume, Monte Carlo 


\section{Introduction}

In the past several decades, polymer-filler composites have attracted great interest from academia and industry because composite materials combine the advantages of each component and can improve the bulk properties significantly. Polymers are useful as a matrix material since they are easy to fabricate, have reasonable strength and durability, and are inexpensive. Blending a small amount of highperformance filler material into the polymer as a dispersed phase can promote properties like gas separation performance, thermal and electric conductivity, and mechanical strength. In the application of gas separation using dense polymeric membrane materials, the performance of traditional polymeric membranes is limited by the trade-off between permeability and selectivity as shown by Robeson in the upper bound plot ${ }^{[1,2]}$. One promising way to improve the gas separation properties beyond this limit is by adding highly permeable and/or selective filler particles such as zeolites and MOFs to improve the gas separation performance above the Robeson upper bound ${ }^{[3-6]}$. Other applications of polymer/filler composites include carbon or silica filler particles dispersed into a rubber matrix to reinforce tires. This can be used to achieve a very high Young's modulus and an exceptional ability for stress transfer, which extends the lifetime of tires dramatically ${ }^{[7]}$. Also, many modern electronic products are made of polymer composites with nano-fillers such as single wall carbon nanotubes (SWCNTs) and silver nanorods, which form conductive networks in the polymer matrix ${ }^{[8,9]}$. The biggest challenge in realizing the full potential of composite materials stems from non-ideal interfacial contact between the polymer and filler particle components. Weak binding between the polymer matrix and filler particles causes void structures and particle agglomeration ${ }^{[10]}$, which decreases properties such as the gas selectivity in membranes. By contrast, strong binding can also generate densified layers around the particle surfaces ${ }^{[10]}$, which results in reduced elasticity and reduction of the gas permeability for membranes. In practice, it is not necessary to eliminate all of the non-ideal interfacial phenomena. In some cases, utilizing non-ideal contact can promote better performance of the materials. For example, it is helpful to create voids at the interface for membranes with high selectivity but low permeability or to have densification at the interface for 
membranes with low selectivity but high permeability. Interfacial morphologies including void structures and polymer densification occur at the nanoscale, which are difficult to observe experimentally. Transmission electron microscopy (TEM) and wide-angle X-ray scattering (WAXS) have been used to this end ${ }^{[11]}$, but TEM reveals only the local structures, while WAXS analyzes the dispersion over a larger volume of a sample.

Molecular modeling and simulation provide a unique way to demonstrate the relationship between the molecular interaction and structure-property relationship of composite interfaces at the nanoscale. However, the relaxation process of polymer chains is extremely slow and the relaxation time $(\tau)$ increases nonlinearly with the chain length in condensed phase; e.g. $\tau$ of a chain with 10 segments is about $10 \mathrm{~ns}$ and $\tau \propto L^{3-3.4[12]}$. Therefore, it is difficult to achieve an equilibrium state using atomistic modeling when starting from a random state. Because of this, most atomistic simulations for polymers in condensed phase will start from the desired equilibrium state that is determined by molecular mechanics ${ }^{[13]}$. This method is not feasible for the polymers in confined spaces or in contact with an inhomogeneous surface, such as composites. In this study, a coarse-grained model has been employed to simulate the phase transition of polymer melts successfully. In the past decade, many confined polymer microstructures that were predicted by coarse-grained lattice models have been verified by experiments ${ }^{[14-19]}$. Employing lattice Monte Carlo simulation, Termonia predicted the stretching and widening of polymer chains near a nanoparticle surface ${ }^{[20]}$. Gao et al. confirmed the formation of the flattened (pancake-like) morphology of the polymer in the region close to a nanosheet surface using coarse-grained molecular dynamics simulations ${ }^{[21]}$. Furthermore, they predicted the rigidification of the polymer layer on a strongly attractive surface. Recently, Hsu and Binder investigated the adsorption transition of a single grafted chain including the effects of chain stiffness in the lattice Monte Carlo simulation ${ }^{[22]}$. Baumgärtner and Muthukumar observed the distinctive effects of physical and chemical surface roughness on the adsorption of a single polymer chain ${ }^{[23]}$. Desorption of a chain from a rough surface is more difficult than from a flat surface due to the "anchoring" of the polymer chain to the wall. They also predicted that the 
desorption temperature of the polymer decreased proportionally to the number of nonadsorbing sites on the surface. In practice, a small amount of roughness (e.g. at nanoscale) on a particle surface may result in significant changes in the physical properties of composite materials including mechanical strength, electrical conductivity and gas separation characteristics.

Venna et al. modified the surface of UiO-66- $\mathrm{NH}_{2}$ based MOF particles to improve their interaction with Matrimid $^{\circledR}$ polymer ${ }^{[5]}$. Various pendant groups were attached to the UiO-66- $\mathrm{NH}_{2}$ MOFs in order to increase the attraction between the filler particle and the polymer while reducing the occurrence of void defects at the interface. The pendant groups tested included phenyl acetyl amide, C10 amide, succinimde, and the unfunctionalized $\mathrm{UiO}-66-\mathrm{NH}_{2}$. It was hypothesized that the phenyl acetyl functional group provided the most attraction to the polymer because it showed the greatest increase in $\mathrm{CO}_{2}$ permeability compared with the neat Matrimid ${ }^{\circledR}$ polymer. However, this behavior can also be explained by the existence of interfacial voids at the interface, and is in fact more likely when the increase in permeability is very large. The rigid benzene pendant structure of the phenyl acetyl group prevents Matrimid ${ }^{\circledR}$ from conforming to the MOF surface and negates any increase in attractive sites between the polymer and particle. By contrast, the $\mathrm{C} 10$ functionalization showed a more modest improvement in $\mathrm{CO}_{2}$ permeability compared with the neat polymer, but this is more consistent with the predictions of the Maxwell model for the maximum attainable increase in $\mathrm{CO}_{2}$ permeability for a mixed matrix membrane with an ideal interface. $\mathrm{C} 10$ consists of a long alkyl chain that is flexible and has a greater ability to intertwine with Matrimid $^{\circledR}$. Clearly the factors that influence the interface morphology are complex and require further investigation. Nevertheless, the interfacial properties of condensed polymers on rough surfaces have not been discussed intensively and most research still focuses on polymer adsorption on flat surfaces.

In this work, we have analyzed polymer composite design parameters including particle surface roughness, polymer-surface interaction, chain flexibility, and chain length to analyze their effects on morphology at the interface using coarse-grained lattice Monte Carlo simulations. Both void structures 
and densification have been demonstrated at the interface under certain conditions, and polymer chain conformations have been quantitatively analyzed.

\section{Methods}

\subsection{Model and Computation}

In this work, we employed a cubic lattice coarse-grained model and developed a Monte Carlo algorithm combining vacancy diffusion ${ }^{[24]}$ and bond fluctuation ${ }^{[25]}$, which have been successfully applied in the prediction of a variety of nanostructures of block copolymer melts ${ }^{[15,17-19]}$. The algorithm of vacancy diffusion and bond fluctuation resulted in much faster equilibration of dense polymer membranes than conventional displacement of polymer beads with fixed bond length. To include the chain rigidity, a bending potential of $\varepsilon_{B}=k_{B}(1-\cos \theta)$ has been introduced, in which $k_{B}$ is the bending potential coefficient and $\theta$ is the angle between two subsequent bond vectors along the chain ${ }^{[22]}$. The non-bond interaction energies have been counted between the nearest neighbor non-bonded sites of polymer beadpolymer bead $\left(\varepsilon_{p p}\right)$, vacancy-vacancy $\left(\varepsilon_{v v}\right)$, polymer bead-vacancy $\left(\varepsilon_{p v}\right)$, polymer bead-surface $\left(\varepsilon_{p s}\right)$, and vacancy-surface $\left(\varepsilon_{v s}\right)$. The attempted motions were limited by excluded volume, allowed bond lengths of 1 and $\sqrt{2}$, and prohibition of bond-crossing. The traditional Metropolis criterion was employed to select the acceptance or rejection of each attempted motion. Every simulation was pre-equilibrated by random self-avoiding moves with all interaction potentials of zero for $5 \times 10^{6}$ Monte Carlo steps multiplied by the number of vacancies. Thereafter, the pre-equilibrated systems were simulated with the full interaction potential for $1.5 \times 10^{7}$ Monte Carlo steps multiplied by the number of vacancies.

In this work, the XYZ dimensions of the systems were set up as $64 \times 64 \times 100$ lattice sites. Two parallel surfaces have been placed outside of the lattice at the Z-direction (Figure 1). Both flat surfaces and surfaces decorated with pendant groups (referred to here as brushes) of 3 lattices long have been studied. 
The volume fractions of polymers were 0.881 between the flat surfaces, and 0.884 and 0.895 between the brush-like surfaces, separately. The effects of the surface density of brushes on the filler surface have been studied. Polymers with chain lengths of 10 and 60 monomers have been included in the simulation.

(a)
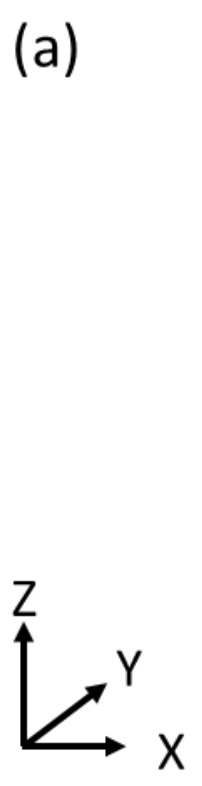

(b)

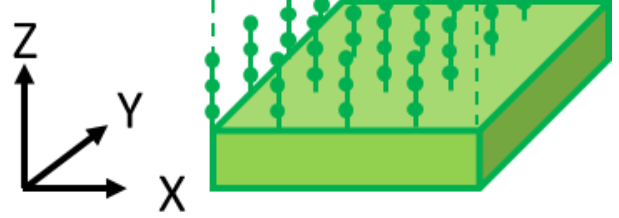

Figure 1. Schematic representation of the confined systems with two parallel surfaces

\subsection{Analysis}

The analysis included the density and fraction of free volume distribution to demonstrate the evolution of interfacial structures under a variety of conditions. The average polymer density distributions along the Zdirection have been calculated:

$\rho_{\text {polymer }}(z)=\frac{1}{N} \sum_{n=1}^{N} \sigma(z, n)$

where $\sigma(z, n)$ is the instantaneous polymer density of the $n t h$ snapshot at position $z$, and $N=500$ is the number of total sample snapshots. 
The fraction of free volume distributions $F F V(z)$ can be computed by:

$F F V(z)=1-\rho_{\text {polymer }}(z)-\rho_{\text {brush }}(z)$

where $\rho_{\text {brush }}(z)$ is the density of the brush structures at position $z$.

When the interaction between surface and polymers is attractive, densified layers may be formed on the surface. It has been reported that the polymer chains in densified layers would be deformed into mushroom like structures, in which the extended conformation occurred in the plane parallel to the surface while the compacted conformation occurred in the perpendicular direction. To quantitatively measure the deformation, an index $(D(z))$ along the $Z$ direction for the chain conformation has been calculated:

$D(z)=\frac{1}{R_{z}(z)} \sqrt{\left(R_{x}^{2}(z)+R_{y}^{2}(z)\right)}$

where $D(z)$ represents the deformation of the chains, of which the mass centers are at position $z$, and

$R_{i}^{2}(z)=\frac{1}{N} \sum_{n=1}^{N} \frac{1}{M} \sum_{m=1}^{M} \frac{1}{L} \sum_{l=1}^{L}\left[i_{l, m, n}(z)-i_{m, n, \text { mean }}(z)\right]^{2}$

where $L$ is the number of the beads per chain; $M$ is the number of chains with mass centers at position $z ; N$ is the number of the total sample snapshots; $i_{l, m, n}(z)$ is the position of $l$ th bead of $m$ th chain of $n$th snapshot at $i$ direction; and $i_{m, n \text { mean }}(z)$ is the mean position of $m$ th chain of $n t h$ snapshot at $i$ direction.

The gyration radius $R_{g}$ of polymer in bulk has also been calculated as shown below in order to study the relationship between interfacial conformation and the ratio of distance from the surface to the overall polymer size.

$R_{g}=\frac{1}{N} \sum_{n=1}^{N} \frac{1}{M} \sum_{m=1}^{M}\left[\frac{1}{L} \sum_{l=1}^{L}\left(r_{l}-r_{\text {mean }}\right)^{2}\right]^{1 / 2}$ 
where $r_{l}$ is the position of the $l$ th bead in the $m$ th chain of the $n$th sample and $r_{\text {mean }}$ is the mean position of beads in $m$ th chain of the $n$th sample.

\section{Results and Discussion}

The density distributions of polymers (chain length $N=60$ ) from the filler surface to the polymer bulk have been calculated and plotted as $\rho$ (density, in this case the ratio of polymer occupied sites/total sites) vs. $Z$ (the dimensionless length representing the number of lattices along the $Z$-direction) and are shown in Figure 2. In order to focus on the structures close to the interface, only the data between $1 \leq Z \leq 6$ have been presented in the following discussion. The polymer density distribution at $95 \leq Z \leq 100$ is symmetrical to $1 \leq Z \leq 6$. The data at $7 \leq Z \leq 94$ has not been presented because the change of the polymer density distribution is very small in this region. Three different surface morphologies have been investigated in this work. They are a smooth plane surface (Figure 2a), a rough brush surface with brush height of 3 lattice units and brush density of 1/16 (6.25\% of the filler surface is occupied by the brushes, Figure $2 b$ ), and a rough brush surface with brush height of 3 lattice units and brush density of 1/4 (25\% of the filler surface is occupied by the brushes, Figure 2c). Polymers with three levels of flexibility were modeled by changing the bending potential coefficient $k_{B}=5.0,2.5$ and $0 k T$, which represent rigid, moderately rigid, and soft chains, respectively. Further, polymers have been confined between filler surfaces where five levels of polymer-filler interaction were studied $\varepsilon_{p s}=-0.5,-0.1,0,0.1$ and $0.5 k T$, which represent strongly attractive, weakly attractive, neutral, weakly repulsive, and strongly repulsive, respectively. The brush beads and the plane surfaces have the same levels of interaction with the polymer. The interaction energies of polymer bead-polymer bead $\left(\varepsilon_{p p}\right)$, vacancy-vacancy $\left(\varepsilon_{v v}\right)$, polymer beadvacancy $\left(\varepsilon_{p v}\right)$, and vacancy-surface $\left(\varepsilon_{v s}\right)$ have been set to 0 . 
Since only short-range interaction between nearest neighbor sites has been included in the non-bond interaction in this lattice model, the degree of surface interaction dominates the polymer density significantly in the region close to the surface $(Z=1$ and 2$)$, but the effects of the surface interaction decreases further away from surface. In the case of a plane filler surface (Figure 1a) with a strong attraction to the polymer, the polymer densifies around the surface as shown in Figure 2a. The normalized density of the polymer around the surface is about 1 , which is much higher than the normalized bulk density of the polymer (0.85). After 1 lattice unit, the normalized polymer density stabilizes to around 0.85. By changing the polymer-filler interaction from strong attraction to weak attraction, the densification of polymer around the particle decreases. With a neutral interaction, the density at the surface is the same as the bulk density. When the surface interaction is weak repulsion, the surface density of the polymer becomes slightly lower than the bulk density. Polymer density at the filler surface decreases much more (to $\sim 0.3$ ) in the case of strong repulsion. The chain rigidity only has a minor effect on polymer density in most scenarios, although there is slightly larger effect in the case of strong repulsion between the polymer and filler. This may be because the bending potential coefficient and angle parameters only directly affect the rigidity of the nearest neighbor sites, and therefore have a short range effect.

Polymers confined between two filler particles with brush-like surfaces (Figure 1b) show different behavior at the interfacial region compared with plane surface particles (Figure 1a). Figure $2 \mathrm{~b}$ represents a composite in which the filler particle has brush-like attachments with a brush density of 1/16 (surface coverage of $6.25 \%$ ). For composites that have strong repulsion between the polymer and filler particle, the polymer density at the interface is much lower than the bulk polymer, although it recovers to the bulk value at lattice distances of $Z>4$. For the weakly repulsive surface, the polymer densities at the interface are also lower than observed with the plane filler. Weakly repulsive composites have a similar trend to strong repulsion, but with higher densities in the interfacial region. In neutral composites, the polymer density is also lower than bulk due to the disruption of polymer chain packing by the brush structures. In 
the attractive surface composites, the polymer densifies at the surface, then decrease in density from $Z=1$ to 3, and returns to bulk density at $Z>4$ (i.e. just beyond the brush region). Increasing the brush density to $1 / 4$ (surface coverage of $25 \%$ ) causes a decrease in polymer density in the brush region since $25 \%$ of sites are occupied by brush structures (Figure 2c). For the strongly repulsive surface, the polymer density is nearly zero at $Z=1$, and becomes approximately equal to the bulk density at $Z>5$, which is two sites away from the end of the brushes. All other levels of interaction have a similar density distribution trend but have higher densities $(\rho=0.5-0.75)$ near the surface and converge to the bulk polymer density at $Z>4$.

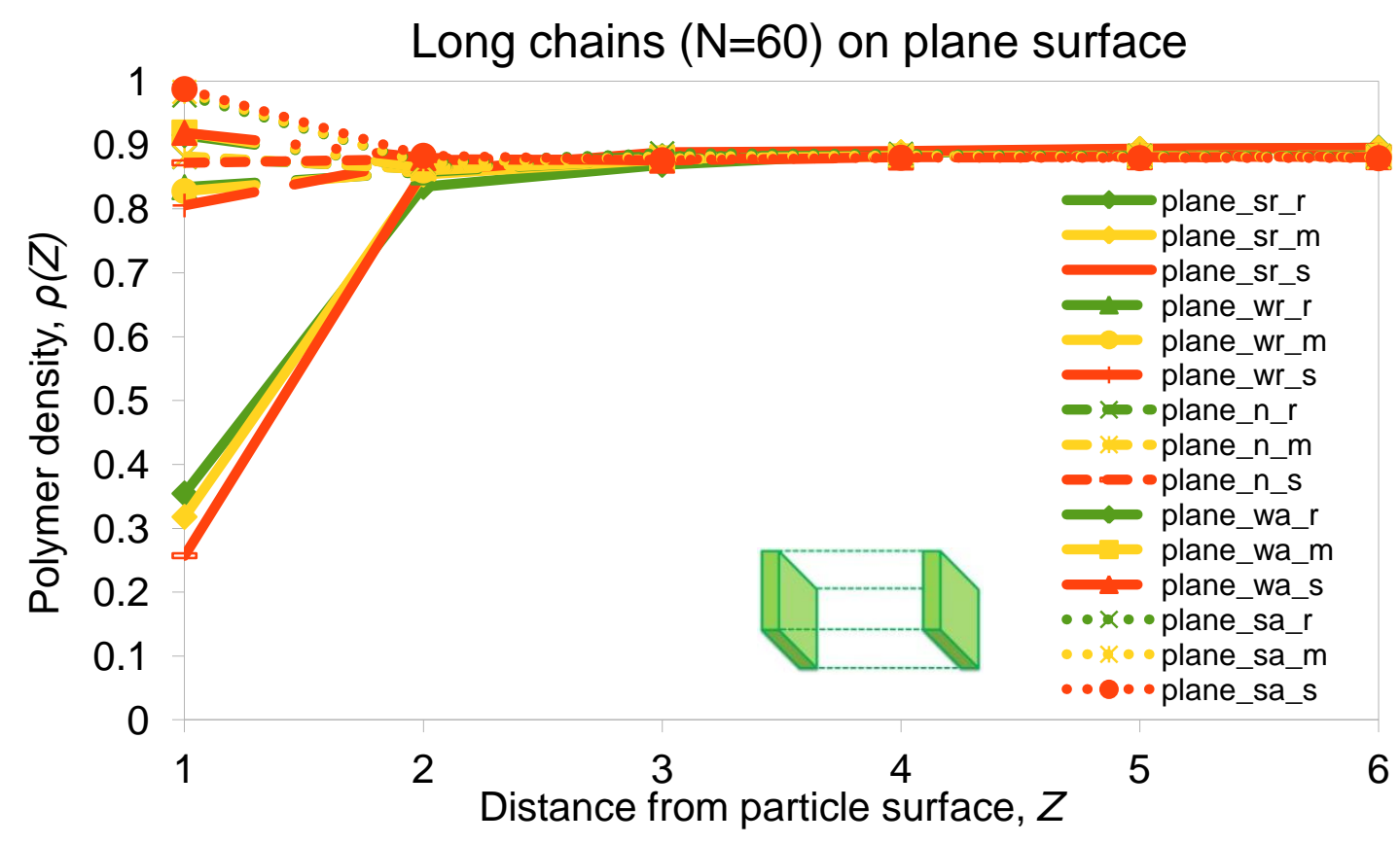

(a) 


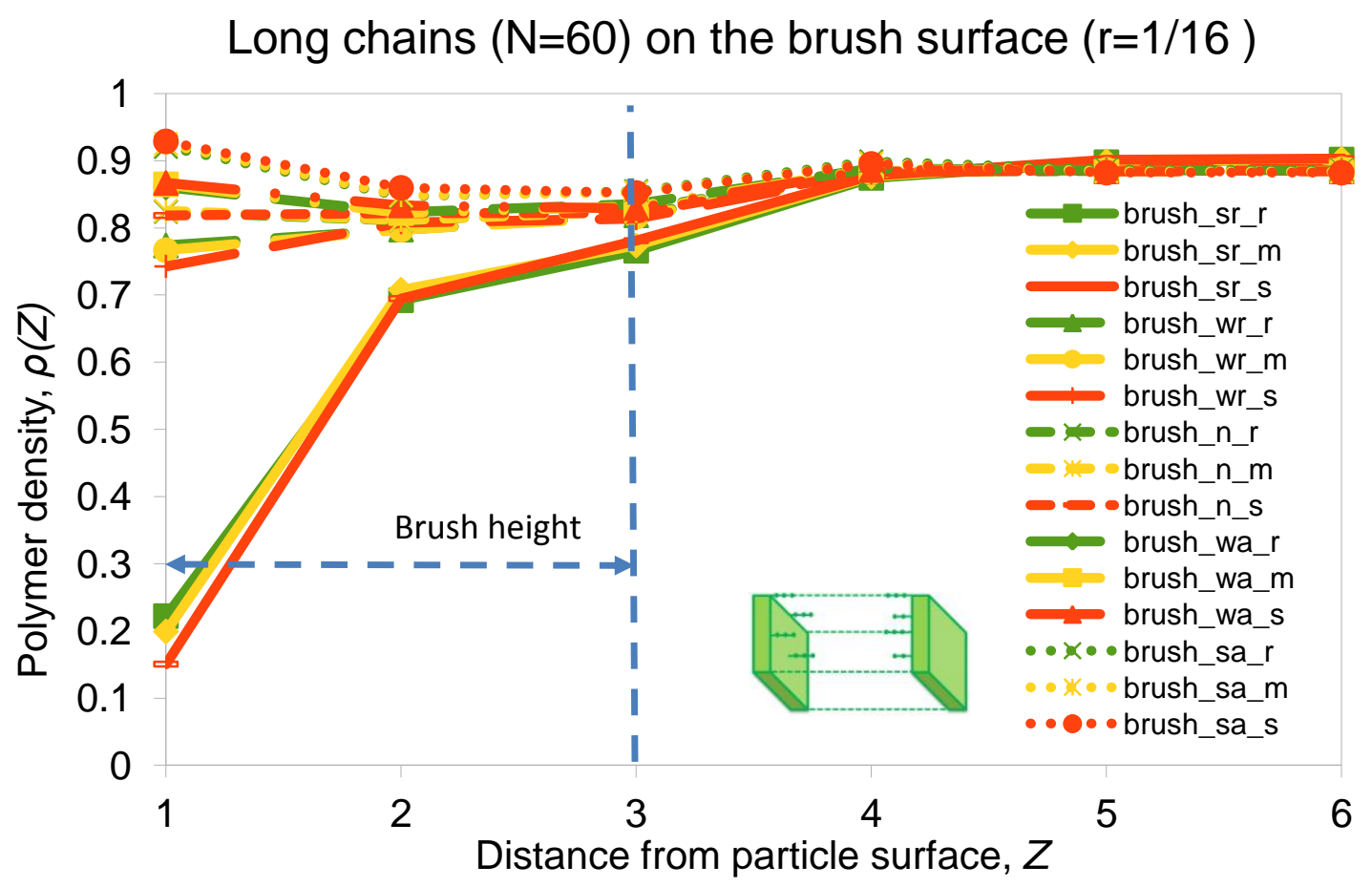

(b)

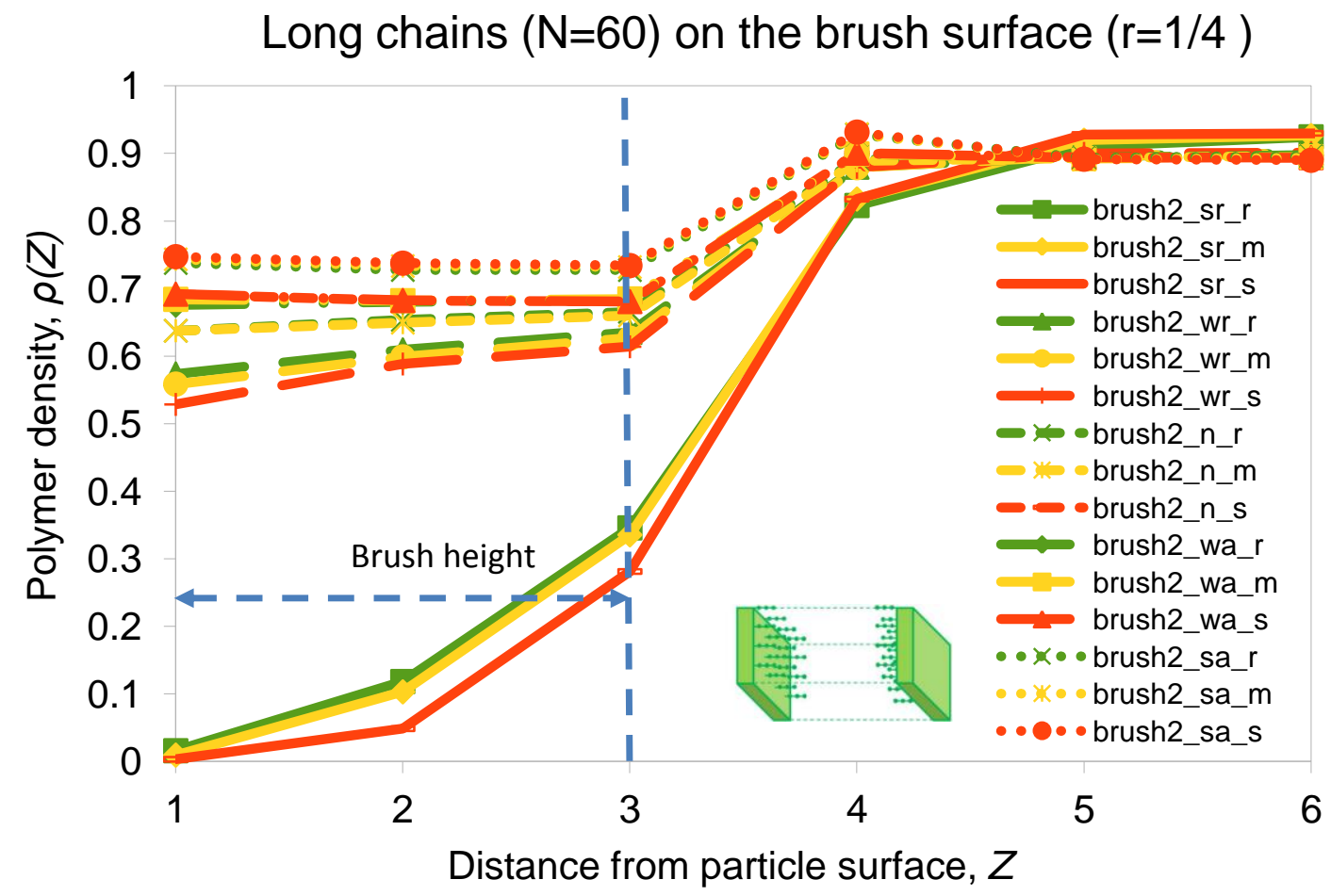

(c) 
Figure 2. Polymer density distributions with a chain length of 60 at the interface of the polymer/filler particle. (a) Filler particle with a plane surface; (b) brush-like surface with brush height of 3 lattices and brush density of $1 / 16$ on the surface; (c) brush-like surface with brush height of 3 and brush density of $1 / 4$ on the surface. Each plot has been labeled as A_B_C, in which A represents surface morphology, B is the surface interaction index and $\mathrm{C}$ represents chain flexibility. $\mathrm{A}=$ plane represents a plane particle surface and $\mathrm{A}=$ brush represents a brush-like surface; $\mathrm{B}=\mathrm{sr}$ means strong repulsion, $\mathrm{B}=$ wr means weak repulsion, $\mathrm{B}=\mathrm{n}$ means neutral, $\mathrm{B}=$ wa means weak attraction and $\mathrm{B}=$ sa means strong attraction; $\mathrm{C}=\mathrm{r}$ represents rigid chains, $\mathrm{C}=\mathrm{m}$ represents moderately rigid chains and $\mathrm{C}=\mathrm{s}$ represents soft chains. The same notation is used in the other figures.

In mixed matrix membranes for gas separation, free volume at the interface is the dominant factor for improving gas permeability and selectivity. A larger free volume generally results in larger permeability but reduced selectivity, and a smaller free volume leads to the opposite results. Weak interaction and a larger gap between the polymer and filler particle can also result in poor mechanical properties or conductivity. Equation (2) was introduced to calculate the fraction of free volume by excluding the sites occupied by brushes and polymers.

The fraction of free volume at the filler surface $(Z=1)$ is affected by the interaction between the polymer and filler particles significantly as shown in Figure 3. In the case of a smooth filler particle surface, strong surface repulsion creates larger fraction of free volume in the vicinity of the nearest neighbor to the surface $(Z=1)$, but strong surface attraction reduces the fraction of free volume dramatically (Figure $3 a)$. However, there are no significant changes in fraction of free volume at $Z>2$ because the surface interaction only has a short range effect. In Figures $3 b$ and $3 c$, in cases of repulsive surface systems, brush structures on the filler can create much larger fraction of free volume at the interface compared with plane surfaces. However, for attractive surfaces, there is smaller fraction of free volume with brush structures 
than in the case of a smooth surface. As with density, the rigidity of the polymer chains plays a minor role in determining the fraction of free volume at the polymer-filler interface.

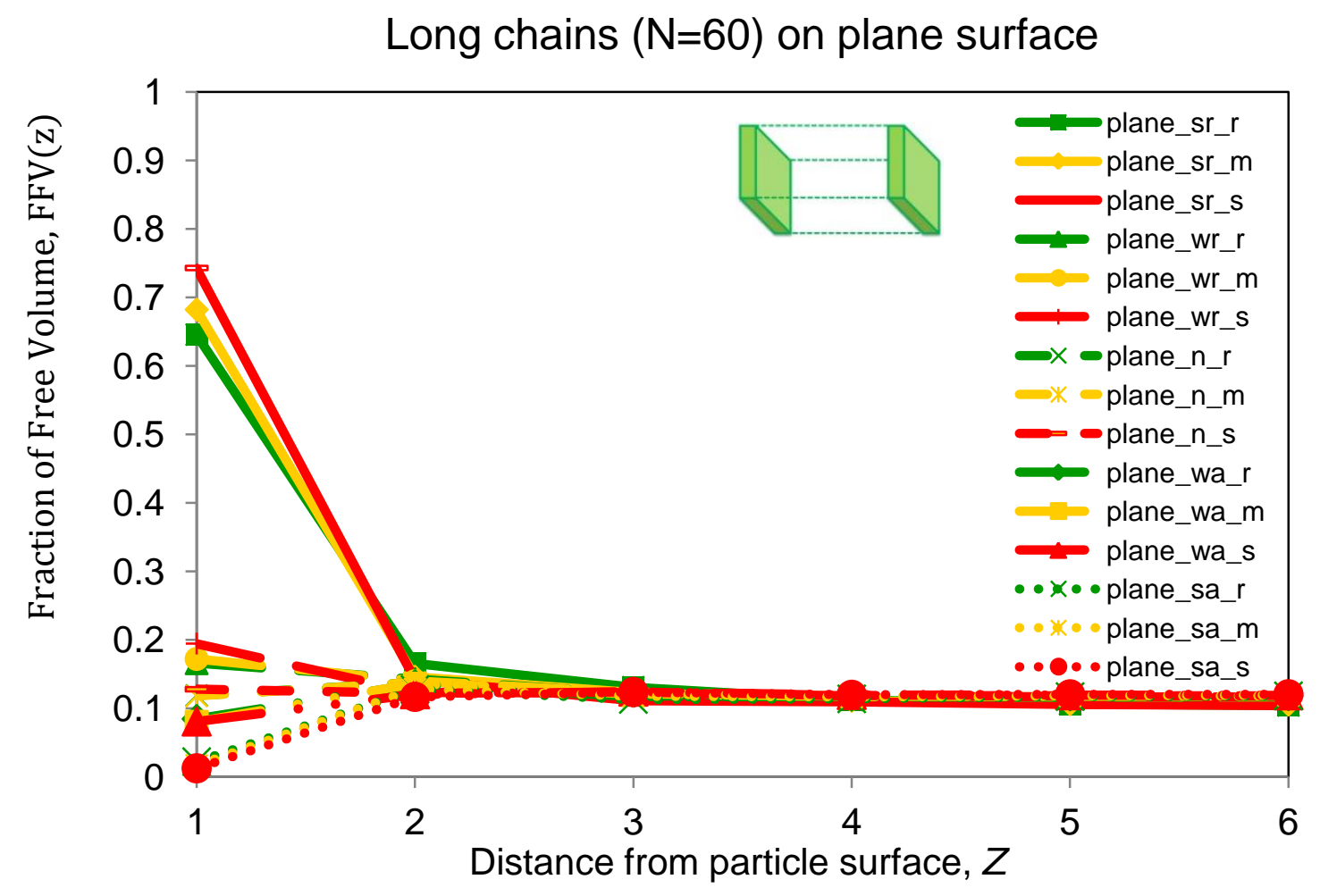

(a) 


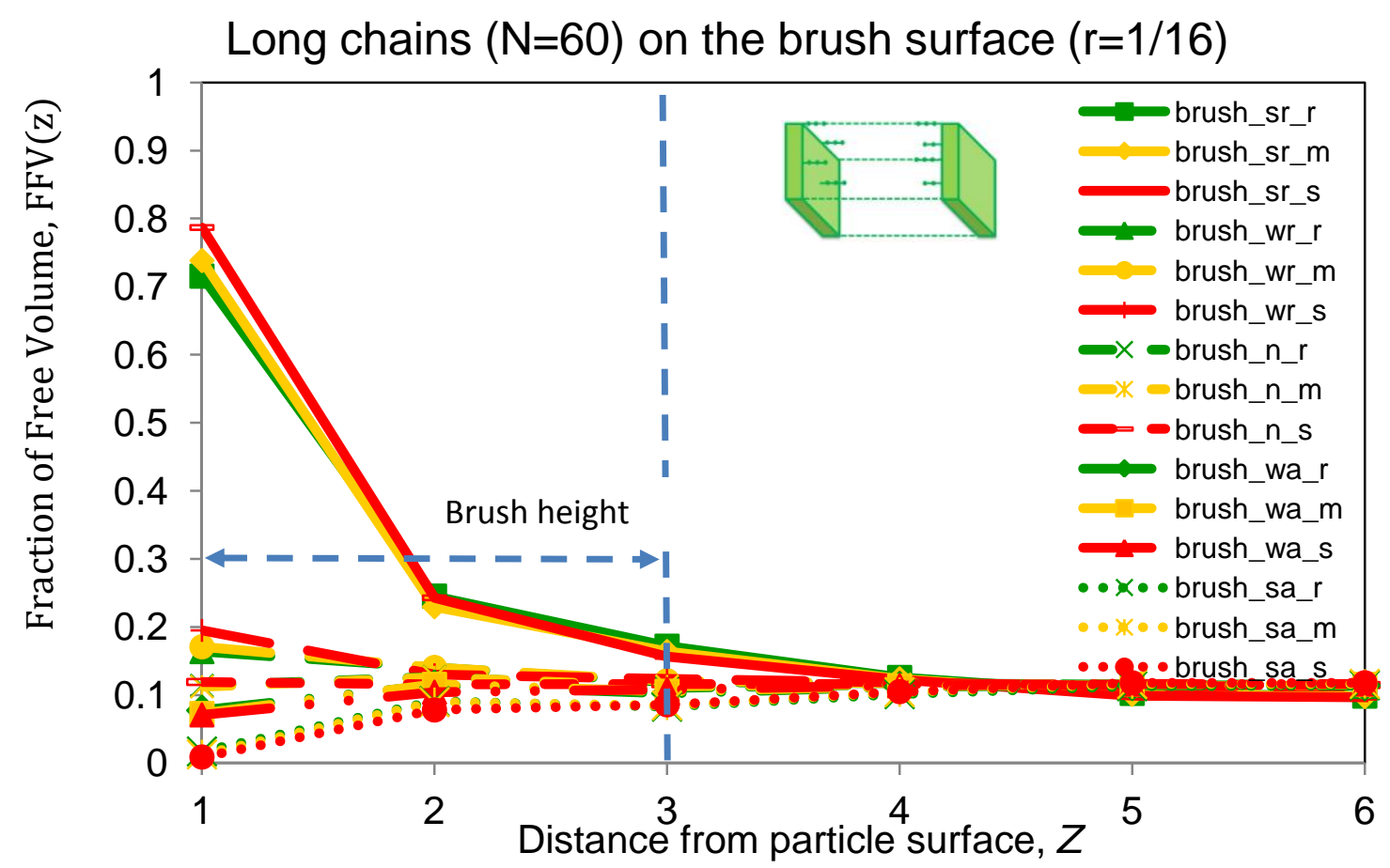

(b)

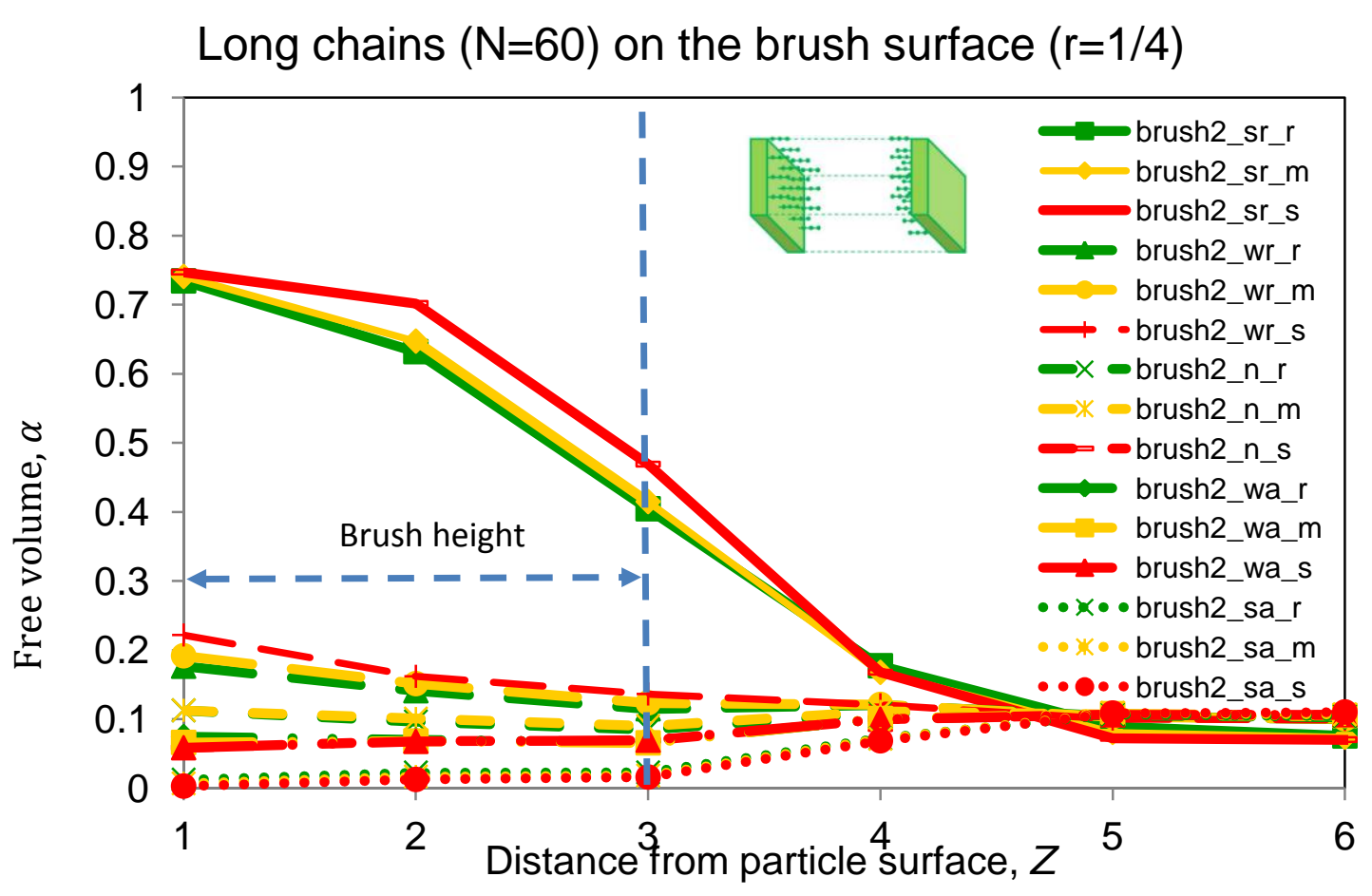

(c) 
Figure 3. Fraction of free volume distributions at the interface between polymer (chain length of 60) and the filler particle surface. (a) Filler particle with a plane surface; (b) brush-like surface with brush height of 3 lattices and brush density of 1/16 on the surface; (c) brush-like surface with brush height of 3 and brush density of $1 / 4$ on the surface.

It has been reported that strong surface attraction can cause polymer chains to densify at the surface and cause the polymer to compress into a flattened "pancake morphology" on the filler particle surface ${ }^{[10]}$. In Figure 4, polymers are more densely packed in the Z-direction when they are closer to the surface for all surface interaction conditions. The deformation index $(D(z)$, Eq. 3$)$ of the polymer is the highest in the case of strong repulsion ( $~ 8$ for rigid polymer chains at the particle surface, $Z=1)$. Changing the rigidity of polymer chains in this model does not significantly affect the results, so only the rigid polymer chain case is shown. For all other polymer-filler interactions such as weak repulsion, neutral, weak attraction and strong attraction, the $D(z)$ profile is the same, with a slightly reduced deformation compared to the strong repulsion case. There is also no significant difference in deformation profiles between the smooth particle surface and the brush-like surface (Figures $4 \mathrm{a}$ and $4 \mathrm{~b}$ respectively). It is interesting that the polymers have more deformation and compression for the strongly repulsive surface than the strongly attractive surface. The mechanism behind this phenomenon can be explained using the cartoon in Figure 5. Polymer chains always suffer structural deformation in close proximity to surfaces due to reduced entropy and less conformational freedom at the interface. For the attractive surface (Figure 5a), the polymer density is high in the region close to the surface so there is limited space for each polymer chain to extend in the plane parallel to the surface. However for the repulsive surface, (Figure 5b) there is more void space at the interface, allowing polymer chains at the interface to fully extend parallel to the surface. These results show that the pancake conformation caused by surface attraction leads to the highest density of polymer chains near the surface, but the most chain deformation occurs due to surface repulsion. The preceding discussion was focused on systems with a polymer length of 60 segments. Shorter polymer chains (10 
segments each) were also simulated for this study and no significant difference in the trends were identified from the long chains. This indicates that chain length plays a very minor role in the polymer/filler interfacial properties.

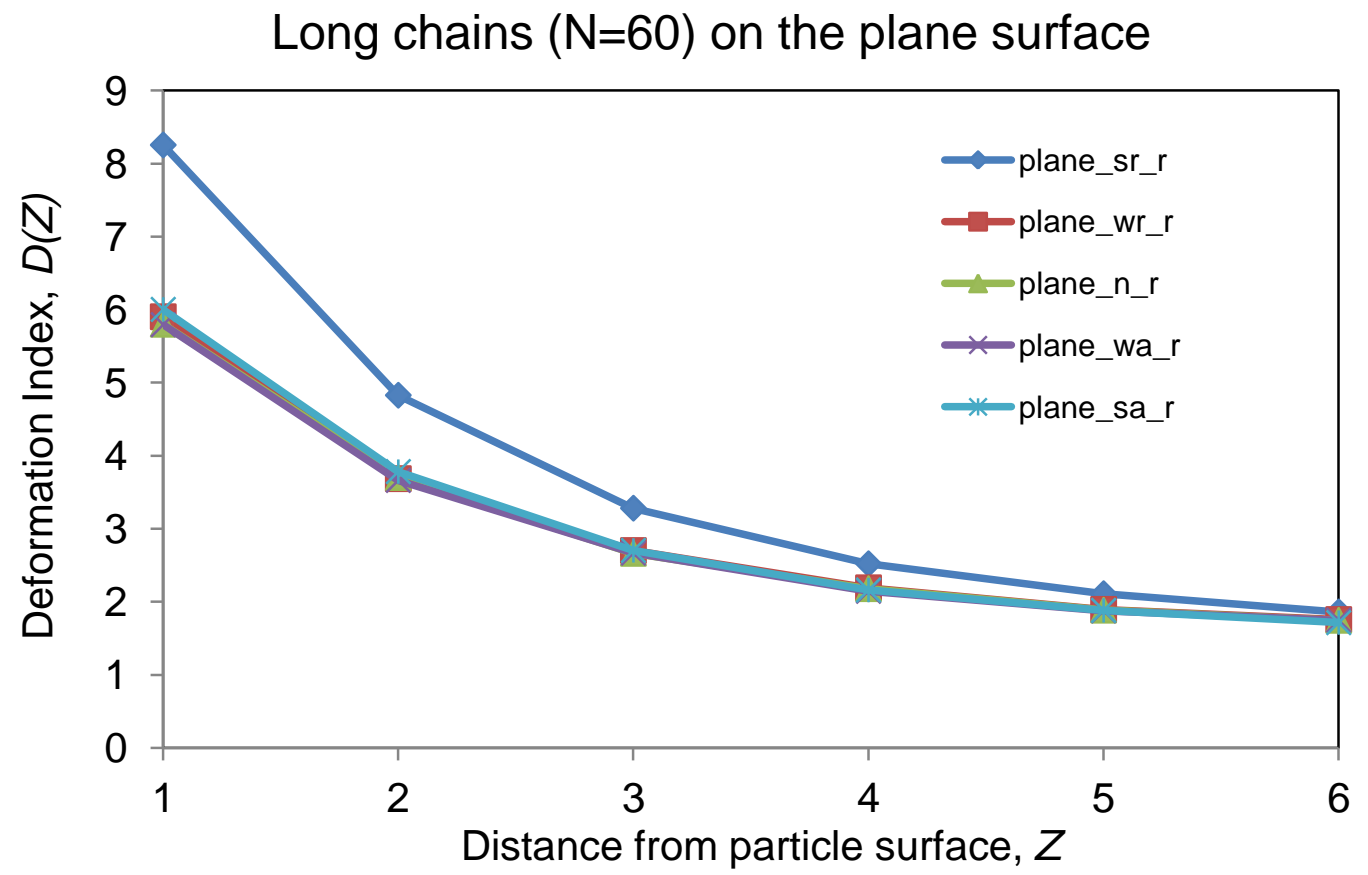

(a) 


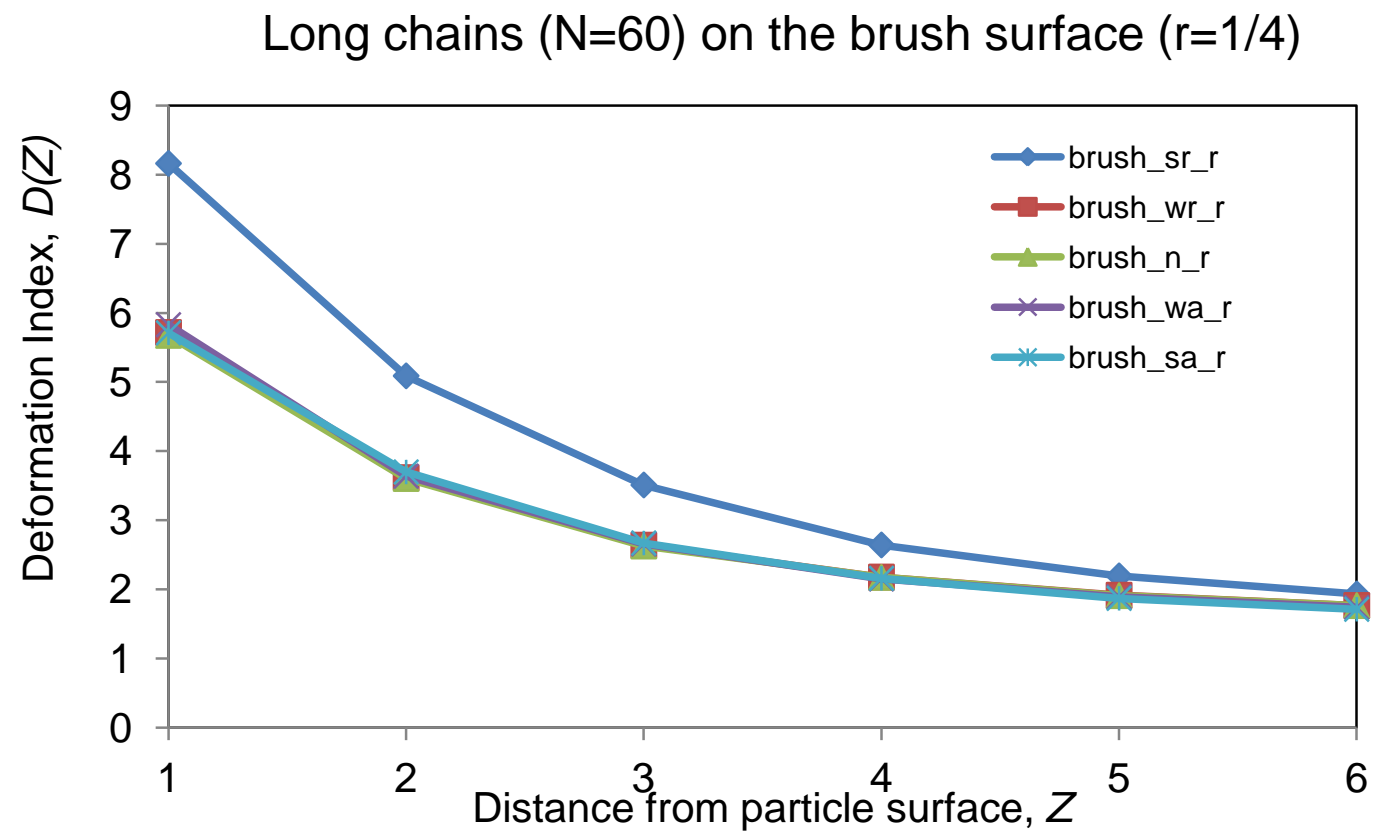

(b)

Figure 4. Deformation index for polymer chains in the direction perpendicular to the filler particle surface.

(a) Smooth surface; (b) brush surface (brush density of 1/4).

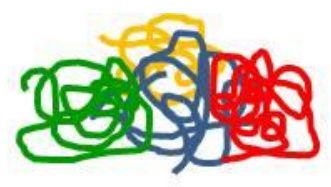

I

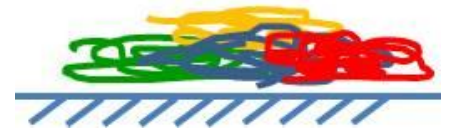

(a)
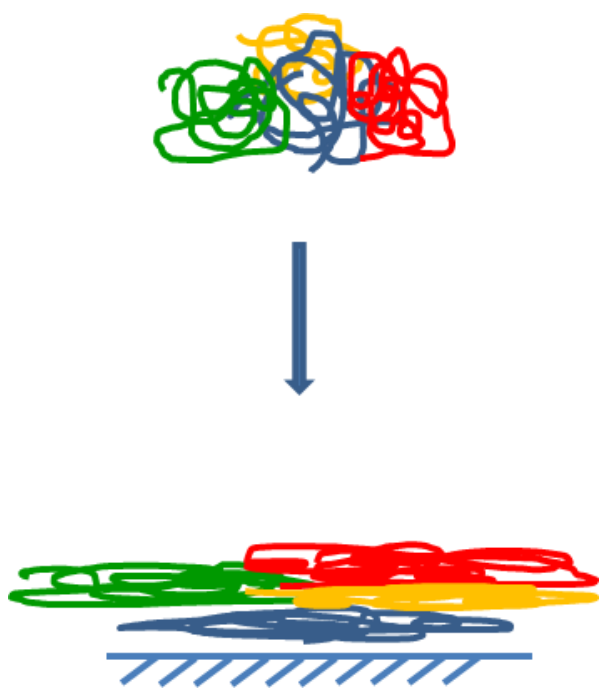

(b) 
Figure 5. Schematic representation of the conformational change of polymer chains in bulk and on a surface for: (a) an attractive surface; (b) a repulsive surface. On a repulsive surface, polymer chains have a slightly more deformation at the surface.

The ratio of the lattice distance $(Z)$ over the gyration radius $\left(R_{g}\right)$ of the polymer is used here to describe the distance from the surface normalized by the size of the molecule. In this work, $R_{g}$ has been calculated using Equation (5) for a polymer with a chain length of 60 lattice spaces, a rigid angle $\left(k_{B}=5.0 k T\right)$ and $R_{g}=4.10$ lattice spaces. The fraction of free volume $(\alpha)$ for a rigid polymer with a strongly repulsive brush surface is shown in Figure 6. As was observed previously in Figure 3, the fraction of free volume is large at the interface but it is reduced to the same level as in the polymer bulk when $Z / R_{g}>1$. As shown in Figure 6, there is no significant change in the distribution of the fraction of free volume when the distance between two surfaces $\left(L_{z}\right)$ is reduced from $L z / R g=24.4$ to $L z / R g=12.2$.

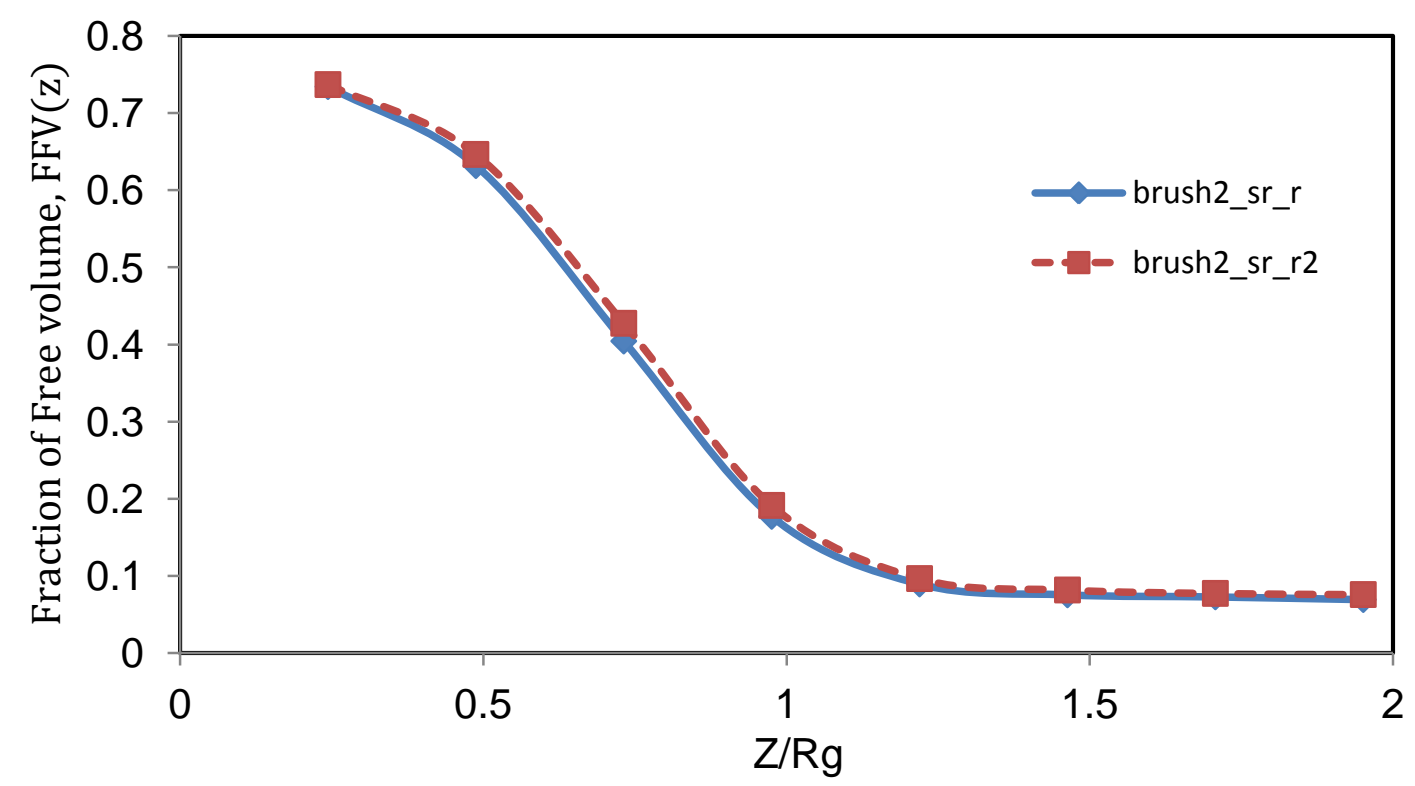

Figure 6. Fraction of free volume distributions at the interface between polymer (chain length of 60) and the filler particle surface. brush2_sr_r: distance between two particle surfaces $L z=100$ lattice spaces $(L z / R g=24.4)$; brush2_sr_r2: distance between two surfaces $L z=50$ lattice spaces $(L z / R g=12.2)$._ 


\section{Summary}

Employing a lattice Monte Carlo simulation, interfacial properties including polymer density distribution, fraction of free volume, and chain conformation have been systematically studied for polymer/filler particle composite structures. It was found that the primary factors influencing these properties are the strength of interaction between polymer/filler particle and the morphology of the filler surface. Changing the interaction of the polymer and filler particle from strongly repulsive to strongly attractive causes the polymer density to increase and the fraction of free volume to decrease in the interface region. In all cases, the most dramatic deviations from the bulk polymer behavior were found in the strongly repulsive systems. Neutrally attractive systems tended to have properties that were most like the bulk polymer, which may or may not be desirable depending on the intended application. Furthermore, contrary to previous reports, polymer chains experienced the most notable flattening or "pancake" deformation were in contact with a repulsive surface rather than an attractive surface. This occurred because a strongly repulsive interaction leads to increased void volume in the interface, and therefore allows more space for polymer chains to extend outwards. There is much larger fraction of free volume at the interface in the polymer/filler pairs that have a repulsive interaction and a brush-like surface rather than a repulsive interaction and a plane surface. Brush-like pendant structures have been attached to particles in other reports in the literature to improve the interaction between the filler particle and polymer. However, from a geometrical perspective, these brushes with sticky structures even create big void space at the interface. Flexibility of polymer chains introduced via the angle potential in the coarse-graining model only slightly influenced the interface structures. In this work, the rigidity among the nearest neighbor segments in a polymer chain have been introduced, but the effect of such rigidity over such a short range was not significant. However, rigidity may become an important factor if it were imposed as a longer range effect and should be investigated in the future. 


\section{List of symbols and abbreviations}

L

$\tau$

$\varepsilon$

$k_{B}$

$\varepsilon_{p p}$

$\varepsilon_{v v}$

$\varepsilon_{p v}$

$\varepsilon_{p s}$

$\varepsilon_{v s}$

$\theta$

$N$

M

$L$

$D(Z)$

$\rho_{\text {brush }}(Z)$

$F F V(Z)$

$\rho_{\text {bead }}$

$i_{m, n, \text { mean }}(Z)$

$I_{l, m, n}(Z)$

$\mathrm{A}=$ plane

$\mathrm{A}=$ brush

$\mathrm{sr}$

wr

$\mathrm{n}$

wa

sa

$\mathrm{r}$

$\mathrm{m}$
Chain length in condensed phase

relaxation time

Polymer chain bending potential

Bending potential coefficient

Polymer bead-polymer bead non-bond interaction energy

Vacancy-vacancy non-bond interaction energy

Polymer bead-vacancy non-bond interaction energy

Polymer bead-surface non-bond interaction energy

Vacancy-surface non-bond interaction energy

Angle between two subsequent bond vectors along the chain

Number of the total sample snapshots.

Number of chains with mass centers at position z;

Number of beads per chain

Chain deformation index

density of brush structures at position $\mathrm{z}$

free volume distributions

average polymer density distributions along Z-direction

Mean position of $m^{\text {th }}$ chain of $n^{\text {th }}$ snapshot at $i$ direction.

Position of $1^{\text {th }}$ bead of $m^{\text {th }}$ chain of $n^{\text {th }}$ snapshot at $i$ direction.

plane surface

brush surface;

strong repulsion,

weak repulsion,

neutral

weak attraction

strong attraction;

rigid chains,

moderate rigid chains 
S

K

$T$

A

soft chains

Boltzmann constant

Temperature

Fraction of free volume

Dimensionless distance from the surface 


\section{Acknowledgements:}

This research was supported in part by an appointment to the National Energy Technology Laboratory Research Participation Program, sponsored by the U.S. Department of Energy and administered by the Oak Ridge Institute for Science and Education. This report was prepared as an account of work sponsored by the Department of Energy, National Energy Technology Laboratory (NETL), an agency of the United States Government, under the NETL Carbon Capture Field Work Proposal. This research was also supported in part by an appointment to the National Energy Technology Laboratory Research Participation Program, sponsored by the U.S. Department of Energy and administered by the Oak Ridge Institute for Science and Education. Neither the United States Government nor any agency thereof, nor any of their employees, makes any warranty, express or implied, or assumes any legal liability or responsibility for the accuracy, completeness, or usefulness of any information, apparatus, product, or process disclosed, or represents that its use would not infringe on privately owned rights. Reference herein to any specific commercial product, process, or service by trade name, trademark, manufacturer, or otherwise does not necessarily constitute or imply its endorsement, recommendation, or favoring by the United States Government or any agency thereof. The views and opinions of authors expressed herein do not necessarily state or reflect those of the United States Government or any agency thereof. 


\section{References:}

1. L. M. Robeson, Correlation of separation factor versus permeability for polymeric membranes, J. Membr. Sci., 62 (1991) 165.

2. L. M. Robeson, Correlation of separation factor versus permeability for polymeric membranes, J. Membr. Sci., 320 (2008) 390.

3. R. Mahajan and W. J. Koros, Mixed matrix membrane materials with glass polymers. part 1, Polym. Eng. and Sci., 42 (2002) 1420; R. Mahajan and W. J. Koros, Mixed matrix membrane materials with glass polymers. part 2, Polym. Eng. and Sci., 42 (2002) 1432.

4. T. Chung, L. Y. Jiang, Y. Li and S. Kulprathipanja, Mixed matrix membrane (MMMs) comprising organic polymers with dispersed inorganic fillers for gas separation, Pro. Polym. Sci., 32 (2007) 483.

5. S. R. Venna, M. Lartey, T. Li, A. Spore, S. Kumar, H. Nulwala, D. R. Luebke, N. L. Rosi and E. Albenze, Fabrication of MMMs with improved gas separation properties using externally-functionalized MOF particles, J. Mater. Chem. A, 3 (2015) 5014.

6. B. Seoane, J. Coronas, I. Gascon, M. E. Benavides, O. Karvan, J. Caro, F. Kapteijna and J. Gascon, Metal-organic framework based mixed matrix membranes: a solution for highly efficient $\mathrm{CO}_{2}$ capture, Chem. Soc. Rev., 44 (2015) 2421.

7. F. Saeed, A. Ansarifar, R. J. Ellis, Y. Haile-Meskel and M. S. Irfan, Two advanced styrenebutadiene/polybutadiene rubber blends filled with a silanized silica nanofiller for potential use in passenger car tire tread compound, J. Appl. Polym. Sci., 123 (2012) 1518.

8. S. Park, M. Vosguerichian and Z. Bao, A review of fabrication and applications of carbon nanotube film-based flexible electronics, Nanoscale, 5 (2013) 1727. 
9. T. C. Hauger, S. M. I. Al-Rafia and J. M. Buriak, Rolling silver nanowire electrodes: simultaneously addressing adhesion, roughness, and conductivity, ACS. Appl. Mater. Interfaces, (5) 2013,12663.

10. T. T. Moore and W. J. Koros, Non-ideal effects in organic-inorganic materials for gas separation membranes, J. Mol. Struct. 739 (2005) 87.

11. J. R. Potts, D. R. Dreyer, C. W. Bielawski, and R. S. Ruoff, Graphene-based polymer nanocomposites, Polymer, 52 (2011) 5.

12. M. Doi, Explanation for the 3.4-power law for viscosity of polymeric liquids on the basis of the tube model, J. Polym. Sci. Part. C. Polym. Lett., 19 (1981) 265.

13. D. N. Theodorou and U. W. Suter, Detailed molecular structure of a vinyl polymer glass, Macromolecules, 18 (1985) 1467.

14. Q. Wang, Q. L. Yan, P. F. Nealey and J. J. De Pablo, Monte Carlo simulations of diblock copolymer thin films confined between two homogeneous surfaces, J. Chem. Phys., 112 (2000) 450.

15. J. Feng, and E. Ruckenstein, The morphology of symmetric triblock copolymer melts confined in a slit: a monte carlo simulation, Macromol. Theory Simul., 11(2002) 630.

16. B. Yu, P. C. Sun, T. H. Chen, Q. H. Jin, D. T. Ding, B. H. Li and A. C. Shi, Confinementinduced novel morphologies of block copolymers, Phys. Rev. Lett., 96(2006) 138306.

17. J. Feng and E. Ruckenstein, Morphologies of ab diblock copolymer melts confined in nanocylindrical tubes, Macromolecules, 39(2006), 4899.

18. J. Feng and E. Ruckenstein, Morphology transitions of ab diblock copolymer melts confined in nanocylindrical tubes, , J. Chem. Phys.,125(2006) 164911. 
19. J. Feng, K. A. Cavicchi and H. Heinz, Control over self-assembly of diblock copolymers on hexagonal and square templates for high area density circuit boards, ACS Nano, 5 (2011) 9413.

20. Y. Termonia, Monte-Carlo modeling of dense polymer melts near nanoparticles, Polymer, 50(2009) 1062.

21. Y. Gao, J. Liu, L. Zhang and D. Cao, Existence of a glassy layer in the polymer-nanosheet interface: evidence from molecular dynamics, Macromol. Theory Simul., 23 (2014) 36.

22. H. Hsu and K. Binder, Effect of chain stiffness on the adsorption transition of polymers, Macromolecules, 46 (2013) 2496.

23. A. Baumgartner and M. Muthukumar, Effects of surface-roughness on adsorbed polymers, J. Chem. Phys., 94 (1991) 4062.

24. J. Reiter, T. Edling and T. Pakula, Monte Carlo Simulation of Lattice Models for Macromolecules at High Densities. J. Chem. Phys., 93 (1990) 837.

25. R. G.Larson, L. E. Scriven, and H. T. Davis, Monte Carlo Simulation of Model AmphiphileOil Water Systems, J. Chem. Phys., 83(1985) 2411. 